
allemande

51-1 | 2019

La République démocratique allemande dans l'espace public européen (1949-2018)

\title{
Schulwissen über die DDR in Frankreich
}

Franziska Flucke et Marie Müller-Zetzsche

\section{(2) OpenEdition \\ Journals}

Édition électronique

URL : https://journals.openedition.org/allemagne/1594

DOI : 10.4000/allemagne. 1594

ISSN : 2605-7913

Éditeur

Société d'études allemandes

Édition imprimée

Date de publication : 2 juillet 2019

Pagination : 177-191

ISSN : 0035-0974

\section{Référence électronique}

Franziska Flucke und Marie Müller-Zetzsche, „Schulwissen über die DDR in Frankreich“, Revue d'Allemagne et des pays de langue allemande [Online], 51-1 | 2019, Online erschienen am: 02 Juli 2020, abgerufen am 02 Juni 2022. URL: http://journals.openedition.org/allemagne/1594 ; DOI: https:// doi.org/10.4000/allemagne.1594 


\section{Schulwissen über die DDR in Frankreich}

\section{- Franziska Flucke*, Marie Müller-Zetzsche **}

Die Frage nach den französischen Deutschlandbildern ist ein beliebtes Thema in der deutsch-französischen Forschungslandschaft. Die Kenntnis von Identitätszuschreibungen gilt als Teilaspekt der interkulturellen Kompetenz und ist für einen gelungenen Dialog zwischen den Partnern unterschiedlicher Kulturen unabdingbar $^{(1)}$. Schulbücher sind diesbezüglich schon länger im Fokus der Forschung, weil sie als „nationale Autobiographien“ (Wolfgang Jacobmeyer) gesellschaftliche Identitäten und ihre Repräsentationen widerspiegeln. Dabei gilt es zu bedenken, dass Schulbücher Medien sind, die in erster Linie für Lehrerinnen und Lehrer gemacht werden. Schulbuchverlage kommen dem Wunsch ihrer Kundinnen und Kunden nach „Bewährtem“ oft nach. Aus diesem Grund setzen sich neue Perspektiven anhand neuer Dokumente vor allem dann durch, wenn der Lehrplan radikale Änderungen fordert oder wenn das Engagement einzelner Autorinnen und Autoren für ein Thema besonders groß ist. Am Beispiel Frankreich untersuchen wir, wie sich die Darstellungen der Deutschen Demokratischen Republik (fortan DDR) als zweiten deutschen Staat in den Lehrbüchern im Laufe der 40-jährigen Existenz und darüber hinaus wandelten und fragen

* Promoviert in Deutschlandstudien und Geschichtsdidaktik an den Universitäten Lorraine-Metz und Siegen und Professeure agrégée en allemand in Straßburg.

* Promoviert in Deutschlandstudien und Kulturgeschichte an den Universitäten Lorraine-Metz und Leipzig und freie Mitarbeiterin im Zeitgeschichtlichen Forum Leipzig.

$1 \mathrm{Zu}$ den Darstellungen des Anderen in den deutsch-französischen Beziehungen in deutschen und französischen Lehrbüchern vgl. u.a. Stéphanie Kra ротн, France - Allemagne. Du duel au duo, de Napoléon à nos jours, Toulouse, Éditions Privat, 2005. Zum Deutschlandbild französischer Jugendlicher vgl. Dieter Josef SснміDт, Bilder vom Nachbarn. Zum Deutschlandbild französischer Jugendlicher, Saarbrücken, universaar, 2014 sowie Franziska Flucke, „Land der Dichter und Denker oder sozialistisches Paradies? Die DDR im Spiegel französischer Deutschlehrbücher (1949-1989)“, in: DIEs. u.a. (Hg.), Der Kalte Krieg im Schulbuch, St. Ingbert, Röhrig Universitätsverlag (= Historica et Didactica. Forschung Geschichtsdidaktik 2), 2017, S. 189-218 und Marie Müller-Zetzsche, „Der Kalte Krieg ist vorbei. Historisierung und Verortung des geteilten Deutschlands in den deutschen und französischen Geschichtsbüchern nach 1990“, in: ebd., S.219-237. 
nach den Erklärungen dafür. Wir untersuchen somit einerseits die Positionierung der Lehrbuchautoren Frankreichs gegenüber der DDR und andererseits - während ihrer Existenz - den Versuch der DDR, auf dessen Fremdbild in Frankreich Einfluss zu nehmen. Anschließend geben wir einen Einblick in die Verortung der DDR als Teil einer transnationalen Erinnerung nach 1989 in einer europäischen Perspektive.

\section{Die Positionierung französischer Lehrbuchautorinnen und -autoren in Deutschbüchern bis 1989}

Während der Existenz der DDR sind je nach kulturpolitischem Kontext verschiedene Positionierungsstrategien gegenüber diesem zweiten deutschen Staat auszumachen. Die vier Lehrbuchgenerationen lesen sich als Spiegelbild der sich wandelnden Beziehungen.

\section{Phase eins: Die politische Inexistenz}

Als sich die deutsch-französische Schulbuchkommission der „Gewerkschaft für Erziehung und Wissenschaft“(2) und der "Fédération de l'Éducation Nationale“ in den 1950er Jahren auch mit den Lehrbüchern der Fremdsprachen beschäftigte, kritisierten die Kommisionsteilnehmer die Überbewertung des Einflusses der Romantik auf das deutsche Geistesleben ${ }^{(3)}$. Sie forderte ein aktuelles Deutschlandbild, das die beiden entstandenen Deutschlands repräsentieren solle ${ }^{(4)}$. Doch bis Ende der 1960er Jahre, die Zeit der diplomatischen Nichtanerkennung seitens Frankreichs ${ }^{(5)}$, spielte der ostdeutsche Staat in den französischen Lehrwerken eine sehr marginale Rolle ${ }^{(6)}$. Thüringen wurde als „grünes Herz Deutschlands“ beschrieben, die Wartburg galt als „Hochburg des deutschen Geistes“ und Weimar war die Heimat von Friedrich Schiller und Johann Wolfgang von Goethe. Die Teilung Deutschlands und die Existenz der DDR wurden den jungen Französinnen und Franzosen vorenthalten. Es dominierte

2 Die „Gewerkschaft Erziehung und Wissenschaft“ (GEW) ist die größte Bildungsgewerkschaft in der Bundesrepublik Deutschland. Sie ging 1948 aus dem „Allgemeinen Deutschen Lehrer- und Lehrerinnenverband“ hervor. Die meisten Mitglieder waren Volksschullehrerinnen und -lehrer, daneben nur wenige Hochschullehrerinnen und -lehrer, meist aus den Pädagogischen Hochschulen wie der Schulbuchspezialist Georg Eckert. Die GEW und die FEN griffen in ihrer bilateralen Schulbuchkommission die gemeinsame Arbeit der Schulbuchrevision der Zwischenkriegszeit auf, um darüber einen Beitrag zur Völkerverständigung und Friedenserziehung zu leisten.

3 Vgl. Franziska Flucke, Bilingualer Geschichtsunterricht in Deutschland und Frankreich. Ein Beitrag $z u$ einem europäischen Geschichtsbewusstsein im Kontext der deutsch-französischen Kulturbeziehungen seit 1945?, Promotion Université Lorraine/ Universität Siegen, Metz, 2018, S. 64.

4 Die Schulbuchprüfer beanstandeten, dass Fotos des Deutschlands nach 1945 oder Werke deutscher Gegenwartskünstler wie Käthe Kollwitz fehlten. Einige der abgedruckten Volkslieder seien „etwas abgedroschen und veraltet“. Rudolf SALEwsky, „Französische Schulbücher in deutscher Sicht“, in: Deutschland und Frankreich im Spiegel ihrer Schulbücher, hg. v. Internationales Schulbuchinstitut an der Kant-Hochschule Braunschweig, Braunschweig, Albert Limbach, 1954, S. 96-99, hier S. 98.

5 Die französische Regierung kannte die DDR erst 1973 als eigenständigen Staat an und war in dieser Hinsicht dem bundesdeutschen Alleinvertretungsanspruch gefolgt.

6 Das heißt aber nicht zwingend, dass aktuelle Ereignisse nicht thematisiert werden. Das „Leitmedium“ Buch macht nur einen Teil der Unterrichtsrealität aus. Vgl. auch Caroline DoubliER, „L'enseignement de l'allemand et image de l'Allemagne depuis la Seconde Guerre mondiale“, Histoire de l'Éducation, 106 (2005), S. 137-152, hier 144. 
weiterhin das Deutschlandbild vom Beginn des 20. Jahrhunderts, das die nationale Mythologie als Ausdruck der „Kollektivseele“ des deutschen Volkes interpretierte. Die Idee vom Nationalcharakter bestimmte die Vorstellungen, so dass die Lehrbücher das Bild des „typischen Deutschen“ entsprach der Darstellung einer allgemeinen Kulturgeschichte wie sie Robert Minder in seinem Werk Allemagnes et Allemands ${ }^{(8)}$ entworfen hatte. Deutschland blieb zunächst ein romantisches, sagenumwobenes Land, in dem Herr Fischer aus Westdeutschland auf die Leipziger Messe fuhr als fände diese in München oder Stuttgart statt $^{(9)}$. Leipzig galt als wohlhabend und nicht von Zerstörungen des Kriegs gezeichnet oder von der Etablierung des Regimes eines neuen totalitären Staats: Herr Fischer besuchte eine moderne und gepflegte Schuhfabrik in der Nähe der Stadt. Die geringen Löhne und die fehlenden unabhängigen Gewerkschaften, kennzeichnend für die wahre Situation der ostdeutschen Arbeiterinnen und Arbeiter in den 1950er Jahren, die nicht zuletzt zum Aufstand vom 17. Juni 1953 geführt hatten, blieben unerwähnt. Dies ist mit der Betonung der sogenannten „klassischen“ Bildung in den Lehrplänen, die noch aus dem Jahre 1938 stammten $^{(10)}$, zu erklären ${ }^{(11)}$. Zudem unterstützte das bundesdeutsche Außenministerium die Verbreitung eines kulturellen und humanistischen Deutschlandbildes, um den Bruch mit dem Nationalsozialismus zu betonen ${ }^{(12)}$. Nicht zuletzt spiegelt das Lehrbuch die Haltung der französischen Öffentlichkeit gegenüber Deutschland wider, war doch in den 1950er Jahren das Interesse an dem zweiten deutschen Staat außerhalb der kommunistischen Kreise nur sehr gering und deren Existenz nur von untergeordneter Bedeutung ${ }^{(13)}$.

7 Die Lehrbuchautoren neigten dabei zu klischeehaften Darstellungen. Sie präsentierten die Bayern als „Säufer der Nation“ und die Niedersachsen waren dagegen „harte Arbeiter" und „gute, blind ergebene Soldaten“. Jürgen Krauskopf, „,Bei Oma saß ein Mann im Kamin...' Neue französische Deutschbücher: Sinnvolles und Ungereimtes“, Dokumente, 38/4 (1982), S.343-350, hier S.343. Vgl. DERS., „Statt Mythen und Sagen - Spiel und Spaß. Deutschbuch im französischen Unterricht - Unkritische DDR-Bilder“, Dokumente, 45/5 (1989), S.382-388, hier S.383; sowie Gilbert Krebs, „L'enseignement de la civilisation allemande. Réflexions et expériences“, in: Robert PicHт, Perspektiven der Frankreichkunde. Ansätze zu einer interdisziplinär orientierten Romanistik, Tübingen, M. Niemeyer, 1974, S. 71-79, hier S. 74.

8 Vgl. Anne KwAschiк, „Relire Allemagnes et Allemands“, Allemagne d'aujourd'hui, nouvelle série 165 (2003), S. 18-34; DIEs., Auf der Suche nach der deutschen Mentalität. Der Kulturhistoriker und Essayist Robert Minder, Göttingen, Wallstein, 2008.

9 L. Bodevin, P. Isler, Collection Deutschland. Stadt und Land. Classes de $5^{e}$, Paris, Masson, 1951.

10 Vgl. C. Doublier, „L’enseignement de l'allemand“ (Anm. 5), S. 145ff. Zu den Rahmenbedingungen des Deutschunterrichts in Frankreich vgl. F. Flucke, „Land der Dichter und Denker“ (Anm. 1), S. $191 \mathrm{f}$.

11 Vgl. Deutschland und Frankreich im Spiegel ihrer Schulbücher (Anm. 4), S.101f.

12 Vgl. Mathias Delori, „L'Office franco-allemand pour la jeunesse. Une contribution au débat sur le poids des idées politiques“, Politique européenne, n 3 (2004), S. 193-201, hier S. 198. Vgl. auch Corine Defrance, Ulrich Pfeil, „Verständigung und Versöhnung. Eine Herausforderung für Deutschland nach 1945“, in: DIEs. (Hg.), Verständigung und Versöhnung nach dem „Zivilisationsbruch“? Deutschland in Europa nach 1945, Bonn, Bundeszentrale für politische Bildung, 2016, S. 21-25.

13 Vgl. Chantal Metzger, „40 ans de relations entre la France et la RDA: du rejet à la reconnaissance officielle (1949-1989)“, in: Dorothee Röseberg (Hg.), Images de la France en République démocratique allemande. Une histoire oubliée, Paris, L'Harmattan, 2004, S. 12-26, hier S. 22 sowie Christian 


\section{Phase 2: Die DDR - ein neuer Staat mit vielen Facetten}

In Folge der Kritik der Lehrbuchkommission und der neuen Lehrpläne Anfang der 1960er Jahre ist zunächst bei den linksorientieren Autoren wie Jean Chassard und Gonthier Weil ein deutlicher Wandel zu beobachten. Die DDR wurde nun aus geographischer, touristischer und wirtschaftlicher Perspektive dargestellt. Somit erhielten die Lernenden erstmals einen multiperspektiven Blick auf den zweiten deutschen Staat ${ }^{(14)}$. Für die Sekundarstufe II setzte sich aber teilweise die Tendenz der 1950er Jahre fort. Der Cours d'allemand für die Première aus dem Jahr 1966 zeigte beispielsweise ein Landschaftsfoto mit zwei Burgen in Mitteldeutschland: die Burg Hanstein im damaligen ostdeutschen Grenzgebiet und die Burg Ludwigstein in Hessen. Auf dem im Westen aufgenommenen Foto kann die Grenze nur vermutet werden ${ }^{(15)}$. Der dazu präsentierte Textauszug verdeckte ebenfalls die Realität der Teilung. So konnte dem Bedürfnis der Lehrkräfte nach Beständigkeit nachgekommen werden. Zugleich thematisierte die Ausgabe für die Abschlussklasse sehr wohl die DDR und deren gesellschaftliche Realitäten. Ein kurzer Autorentext beschrieb die deutsche Teilung als Resultat mangelnden Konsenses zwischen den Westmächten und der Sowjetunion ${ }^{(16)}$. Die gleiche Ausgabe sprach auch ganz selbstverständlich von der „Deutschen Demokratischen Republik“. Daraus können wir schließen, dass die Lehrbuchautoren die DDR bereits vor der offiziellen Anerkennung seitens der französischen Republik als selbstständigen Staat betrachteten und versuchten, das mythische, kulturelle Deutschland mit den neuen Realitäten zusammenzubringen.

Eine Fotographie zeigte zudem die Mauer an der Bernauer Straße, auf der in großen schwarzen Buchstaben „KZ“ geschrieben worden war. Daneben ist folgendes Zitat zu lesen: „Antifaschistischer Schutzwall oder Schandmauer?“ Drei Fragenkomplexe sollen die Lernenden bei der Textarbeit leiten und sie zur Diskussion anregen. Dabei ist besonders die letzte Frage interessant, in der die Autoren den Begriff „freie Welt" als Bezeichnung für den Westen in Anführungszeichen setzten. Mit dieser Wortwahl stellten die beiden Germanisten Jean Chassard und Gonthier Weil diese zumindest teilweise in Frage und trugen somit zur Etablierung der DDR als Alternativmodell im französischen Diskurs bei. Sie positionierten sich damit indirekt als Mitstreiter der ostdeutschen Anerkennungspolitik. Ihr Lehrwerk war in Folge mehrere Jahrzehnte lang eine Referenz bei der Deutschlehrerprüfung, dem CAPES ${ }^{(17)}$, in Frankreich und prägte somit das DDR-Bild mehrerer Lehrergenerationen. Um den innovativen Charakter von Chassard/Weil zu unterstreichen, muss betont werden, dass der Großteil der Lehrwerke dieser Epoche eher dem bundesdeutschen Alleinvertretungsanspruch

Wenkel, „Des images françaises du socialisme en RDA (1956-1969)“, in: Chantal Metzger (Hg.), La République démocratique allemande. La vitrine du socialisme et l'envers du miroir (1949-1989-2009), Brüssel, Peter Lang, 2009, S. 219-231, hier S. 219.

Vgl. J. Krauskopf, „Bei Oma saß ein Mann im Kamin“ (Anm. 7), S. 343.

Vgl. Jean Chassard, Gonthier Weil, Cours d'allemand. Classe de Première, Paris, Colin, 1966, S. 60.

„Mit der Sowjetunion konnten aber die Westmächte zu keiner Einigung gelangen und ein zweiter deutscher Staat, die Deutsche Demokratische Republik, entstand, der von der Bundesrepublik durch den sogenannten ,Eisernen Vorhang' getrennt wurde." Jean CHAssARD, Gonthier Weil, Cours d'allemand. Classes Terminales, Paris, Colin, 1967, S. 92.

17 Das Certificat d'aptitude au professorat de l'enseignement du second degré (kurz CAPES) ist ein „Concours" („Wettbewerb“) für angehende Sekundarschullehrerinnen und -lehrer in Frankreich. 
folgte und die DDR entsprechend völlig ausblendete, wie Jürgen Krauskopf es für „Bilder und Stimmen“ feststellte ${ }^{(18)}$.

\section{Phase 3: Eine Reise in das sozialistische Paradies?}

In den 1970er wandelten sich die Darstellungen erneut. In Folge der diplomatischen Anerkennung 1973 erschien die DDR nun gleichberechtigt neben der Bundesrepublik. Der Bau der Berliner Mauer hatte die Wahrnehmung einer breiten französischen Öffentlichkeit für die Existenz eines zweiten Deutschlands geschärft und ihr paradoxerweise einen flächendeckenden Einzug in die Lehrbücher ermöglicht ${ }^{(19)}$. Die deutsche Teilung fand jetzt in den Kapiteln zur Politik und Wirtschaft Erwähnung ${ }^{(20)}$. Dabei entwarfen auffällig viele Texte aus offiziellen Regierungsbulletins ein positives und idealisiertes Bild der DDR ohne Kontextualisierung. Das Lehrbuch des Elsässer Lehrers und späteren Schulinspektor Georges Holderith ${ }^{(21)}$ veranschaulicht dies sehr deutlich. Er widmete ein ganzes Kapitel der bundesdeutschen Presselandschaft und verschwieg die ostdeutsche Variante. Nur am Ende des Lehrbuchs stellte ein Autorentext die DDR sowie die deutsch-deutschen Beziehungen auf einer Seite kurz vor. Das Lehrwerk stellte ferner Auszüge aus dem Grundgesetz kommentarlos Passagen aus der DDR-Verfassung gegenüber und vermittelte so den Eindruck, die Presse- und Meinungsfreiheit sei auf beiden Seiten garantiert ${ }^{(22)}$. Der diktatorische Charakter wurde - wenn überhaupt - beiläufig erwähnt, die DDR-Propaganda dagegen wortwörtlich übernommen ${ }^{(23)}$. Die DDR war zu einem politischen Konstrukt geworden, über das Leben der Menschen in diesem Staat erfuhren die Schülerinnen und Schüler jedoch kaum etwas. Dies war das Resultat einer gezielten Imagekampagne der DDR in Frankreich. Der Verlag „Zeit im Bild“ veröffentlichte 1971 die Broschüre La R.D.A. se présente. Darin präsentierte sich die DDR mit zahlreichen Farbfotos als einer der führenden Industriestaaten, in dem sich alles um das Wohl der Bürger drehe ${ }^{(24)}$. Außerdem hatte die französische Germanistik 1966 die gegenwartsbezogene Landeskunde als offiziellen dritten Lehrbereich der Germanistik an den Hochschulen eingeführt und kommunistisch orientierte Germanisten und Historiker wie Gilbert Badia oder Georges Castellan waren nun an der Lehrerausbildung beteiligt. Beide setzten sich für ein positives DDR-Bild in Frankreich

18 Vgl. Jürgen Krauskopf, Das Deutschland-und Frankreichbild in Schulbüchern. Deutsche Französischbücher und Französische Deutschbücher 1950-1980, Tübingen, Narr (= Gießener Beiträge zur Fremdsprachendidaktik), 1985, S. 209.

19 Zum Einzug der DDR in die Geographiebücher vgl. Christian Wenkel, Auf der Suche nach einem „anderem Deutschland“. Das Verhältnis Frankreichs zur DDR im Spannungsfeld von Perzeption und Diplomatie, Oldenburg, De Gruyter (= Studien zur Zeitgeschichte, 86), 2014, S. $23 f$.

20 Vgl. J. Krauskopf, „Bei Oma saß ein Mann im Kamin“ (Anm. 7), S. 344-346.

21 Georges Holderith führte 1972 den Deutschunterricht an den elsässischen Grundschulen wieder ein, was als „réforme Holderith“ in die französische Bildungsgeschichte einging. Er entwickelte zudem eine eigene audiovisuelle Unterrichtsmethode, die die besonderen Sprachkenntnisse im Unterricht berücksichtigte. Vgl. Dominique HUCK, „L'enseignement de l'allemand à l'école élémentaire en Alsace. Questions de méthode: les manuels en usage entre 1952 et 1990“, Histoire de l'éducation, 106 (2005), S. 217-267.

23 Vgl. ebd.

24 Herbert Arndt u.a., La R.D.A. se présente, Dresden, Zeit im Bild, 1971, S. 170. 
ein ${ }^{(25)}$. Unter ihrer Leitung widmeten sich Magisterarbeiten und Dissertationen zunehmend der DDR, die für einen Teil der französischen Germanistik „eine Utopie, ein Gegenbild zur Bundesrepublik“ bot ${ }^{(26)}$. Die Biermann-Affäre relativierte dies Idealbild zwar in der Öffentlichkeit, in die Lehrbücher zog eine kritische Haltung gegenüber der DDR aber erst wieder in der nächsten Dekade ein, als auch eine breitere Berichterstattung in den französischen Medien zu beobachten war.

\section{Phase 4: Die DDR als gescheiterte Utopie?}

Ein erneuter Wandel der DDR-Bilder ist in den 1980er Jahren zu beobachten. Quantitativ wie in der Differenziertheit der Darstellung nahm die DDR in den Lehrbüchern nun die gleiche Stellung wie die Bundesrepublik ein. Alle Lehrbücher gaben jetzt auch detailreiche Informationen und präsentierten Auszüge ostdeutscher Autorinnen und Autoren. Diese breitere Perspektive entsprach den veränderten Beziehungen zwischen der DDR und Frankreich nach der Unterzeichnung des Kulturabkommens 1980 einerseits ${ }^{(27)}$ und den Beschwerden des bundesdeutschen Auswärtigen Amtes andererseits. Letzteres war bezüglich der sehr DDR-freundlichen Darstellung im französischen Schul- und Hochschulunterricht besorgt und forderte ein korrigiertes und aktualisiertes Deutschlandbild in den Lehrbüchern ${ }^{(28)}$. Daher wurden nun auch Werke von Christa Wolf, Anna Seghers, Günter de Bruyn oder Christoph Hein, die als „Vorzeigeautoren“ auch in Westdeutschland Verleger fanden, aufgenommen und Themen wie „Republikflucht“ angesprochen ${ }^{(29)}$.

25 Escherich unterteilt die französische DDR-Forschung in drei Strömungen. Die erste Forschergruppe, zu der Badia, ein Verfechter der frühen Anerkennung der DDR und zugleich einer der wichtigsten Mittelsmänner zwischen Frankreich und der DDR, gehörte, zeichnete ein sehr positives Bild der DDR. Eine zweite Gruppe, darunter Castellan, habe die DDR nach deren eigenen Maßstäben beurteilt, d.h. die DDR sei eine Realität gewesen, die es möglichst nüchtern zu beschreiben galt. Castellans Perspektive, nach der der ostdeutsche Staat als vorbildliche Realisierung des Sozialismus zu betrachten sei, fand direkten Eingang in die Geographiebücher Frankreichs. Eine dritte Gruppe um Joseph Rovan und Alfred Grosser stand der DDR auf Grund ihres repressiven Charakters und der Indoktrination ablehnend gegenüber. Vgl. Bernhard Escherich u.a., „Das Bild der DDR in Frankreich bis 1989“, Dokumente, 56/1 (2000), S.25-32, hier S.28, 31f.; Ulrich PfeIL, „Badia, Gilbert“, in: Nicole Colın u.a. (Hg.), Lexikon der deutsch-französischen Kulturbeziehungen nach 1945, Tübingen, Narr, 2015 (2. Aufl.), S. 115-117; C. Wenkel, „Des images françaises“ (Anm. 13), S. 224.

$26 E b d$. sowie Ulrich Pfeil, „Die französische DDR-Forschung vor 1990. Eine analytische Annäherung über ihre Zeitschriften“, in: Michel Grunewald u.a. (Hg.), France - Allemagne au XXe siècle: la production académique de savoir sur l'Autre, IV: Les médias, Bern, Peter Lang, 2014, S. 109-125.

27 Am 12.12.1983 wurde das DDR-Kulturzentrum in Paris eröffnet, womit die DDR fortan auch physisch in Frankreich präsent war und ihr kulturelles Schaffen bewarb. Vgl. Ulrich PfeIL, „Die Rückkehr der gesamtdeutschen Kulturnation. Das DDR-Kulturzentrum in Paris“, Lendemains, 103/104 (2001), S. 108-131.

28 Vgl. Rainer Riemenschneider, „Die dritte Generation. Schulbucharbeit mit Frankreich aus der Sicht eines Akteurs und Zeitzeugen“, in: Rainer Bendick u.a. (Hg.), Deutschland und Frankreich Geschichtsunterricht für Europa. Die deutsch-französischen Schulbuchgespräche im europäischen Kontext / France-Allemagne. L'enseignement de l'histoire pour l'Europe. Les rencontres franco-allemandes sur les manuels scolaires dans le contexte européen, Frankfurt am Main, Wochenschau Verlag, 2018, S. 60-70, hier S. 62.

29 Vgl. Danielle Risterucci-Roudnicky, France - RDA. Anatomie d'un transfert littéraire 1949-1990, Bern, Peter Lang, 1999; Karin R. GüTtLeR, Die Rezeption der DDR-Literatur in Frankreich (1945-1990). 
Besonders deutlich zeigen die Oberstufenlehrbuchreihen Ja, aber (1982) und Meiner Meinung nach (1983) den Wandel. Ja, aber nutzt einen Auszug aus Christa Wolfs Werk Der geteilte Himmel als Diskussionsanlass, um die Teilung der Stadt zu thematisieren ${ }^{(30)}$. Ein weiteres Beispiel ist ein Auszug aus Klaus Schlesingers Erzählung Am Ende der Jugend (1973). Der 1980 nach West-Berlin übergesiedelte Autor schildert die Erlebnisse des 13. August 1961 unbeschönigt $^{(31)}$. Erich Loest berichtet seinerseits in der Erzählung Eine Falte, spinnwebfein (1974) die strenge politische Selektion beim Zugang zur Erweiterten Oberschule, der Abiturstufe der DDR. Auf dem Seitenrand stand ein kurzer Verweis auf das entsprechende Thema in den „Fakten“ “(32). Diese Präsentationsweise offenbarte den Schülerinnen und Schüler zwei Perspektiven auf das Leben in der DDR: Zunächst die offiziellen Fakten und in einem zweiten Schritt die in der DDR-Literatur „versteckte“ Systemkritik.

Die Lehrbuchautoren vermieden so eine persönliche Positionierung und bemühten sich - gemäß dem Grundsatz keine Politik im Unterricht - um absolute Neutralität, wie die historischen Kapitel des Buches zeigen. Landeskundliches und kulturgeschichtliches Wissen wird in „Brennpunkten“ dargestellt. Brennpunkt 8 widmete sich der „Nachkriegszeit und Gegenwart“ und präsentiert das „Potsdamer Abkommen“ als Ausgangspunkt der Spaltung Deutschlands. Im Folgenden wurden dann die Verfassungen und Hymnen - wieder kommentarlos - gegenübergestellt sowie Markt- und Planwirtschaft erklärt. Die anschließende Doppelseite thematisierte den „Währungskrieg“, „Die Berlin-Blockade“ und „unvereinbare Strategien“, die Flüchtlingsfrage, „Der ,Eiserne Vorhang““ und abschließend Schritte der Neuen Ostpolitik mittels kurzer Texte, Fotomaterial und Statistiken ${ }^{(33)}$. Brennpunkt 11 ließ die Vermutung zu, dass die DDR ein pluralistischer Staat mit gleichen Rechten für alle Parteien war. Bei der Vorstellung der politischen Parteien und Massenorganisationen wird an keiner Stelle erwähnt, dass alle Parteien unter Führung der SED standen und die Massenorganisationen deren Positionen als „Transmissionsriemen“ in die Gesellschaft trugen. Augenfällig ist zudem das häufige Zitieren des Dramatikers Bertolt Brecht. Von insgesamt 88 literarischen Texten waren zehn von dem ostdeutschen Lyriker und Dramatiker ${ }^{(34)}$. Er erhielt zudem im Brennpunkt 10 „Dichtung Kunst im 20. Jh. (2)“ eine eigene Seite, die ihn als Kapitalismuskritiker präsentierte. Die Präsenz im Lehrbuch entsprach der

Autoren und Werke im Spiegel der Kritik, Bern, Peter Lang, 2001; Nicole Colin, Deutsche Dramatik im französischen Theater nach 1945: Künstlerisches Selbstverständnis im Kulturtransfer, Bielefeld, transcript, 2011.

30 „Was bedeutet Manfreds Satz: ,den Himmel wenigstens können sie nicht teilen“?“ Jean-Claude Duport u.a., Ja, aber... Terminales Langue 1 et Langue 2, Paris, Colin, 1982, S. 85.

31 Vgl. Astrid KöHLER, Brückenschläge. DDR-Autoren vor und nach der Wiedervereinigung, Göttingen Vandenhoeck \& Ruprecht, 2007, S. 64.

32 Jean-Claude Duport, Ja, aber... Première Langue 1, Paris, Colin, 1981, S. 200.

33 Der Volksaufstand vom 17. Juni 1953 fand nun auch Erwähnung, aber nur über die Bildikone der Pflastersteine werfenden Jugendlichen. Das Bild mit der Legende „17. Juni 1953 Ostberliner Arbeiter lehnen sich gegen die Regierung auf“ stimmte auf die Textgruppe „Mut, Feigheit“ ein. Weitere Erklärungen zu den Ereignissen in Berlin oder zur Herkunft des Fotos fehlten allerdings. Ebd., S. 103.

34 Die zitierten Werke sind Johanna der Schlachthöfe in drei Teilen, Das Leben des Galilei, Der verwundete Sokrates, Kalendergeschichten (drei Mal) sowie Furcht und Elend des Dritten Reichs. Vgl. ebd. 
auf den französischen Theaterbühnen, wo er bis in die 1990er Jahre der meistgespielte deutschsprachige Autor blieb ${ }^{(35)}$.

Erst ganz zum Ende des Lehrwerkes tauchte Kritik an der DDR auf, wieder über die Kunst. Unter der Zwischenüberschrift „Die Demokratie in Frage...“ wurde eine Karikatur der Gitarre von Wolf Biermann präsentiert, die der Karikaturist Gabor Benedek mit Stacheldraht-Saiten bezogen und mit dem Etikett „Produkt der DDR“ versehen hatte. Zwei Zitate unterstrichen nun die Abwesenheit der Meinungsfreiheit ${ }^{(36)}$. Anschließend wurde allerdings der "Radikalerlaß“ von 1972 und das darin festgehaltene „Berufsverbot“ als Anlass für die Frage genutzt, ob die „Die BRD, ein Polizeistaat?“(37) sei. Diese Frage relativierte den autoritären Charakter der DDR und spiegelte die Meinung der französischen Öffentlichkeit wider, die die Haltung der Bundesregierung bezüglich der Roten Armee Fraktion und der "Berufsverbote“ als hart betrachtete ${ }^{(38)}$. Diese Tendenz zur Parallelkritik an Westdeutschland verstärkte sich Ende der 1980er Jahre. In Ja, aber... aus dem Jahr 1989 finden wir wiederholt Ausschnitte aus dem Jugendbuch Treffpunkt Weltzeituhr (1984) von Isolde Heyne, die 1985 den deutschen Jugendliteraturpreis erhalten hatte. Die Autorin, 1979 in die Bundesrepublik übergesiedelt, verarbeitete darin ihre Erlebnisse. Über die Romanfigur Inka lernten die französischen Schülerinnen und Schüler nun auch den Blick der Ostdeutschen auf den Westen kennen. Inka hatte die DDR verlassen und war zunächst mit dieser „neuen“ Welt überfordert. Sie verließ z.B. den Klassenraum weinend, weil sie die „Ossi“-Witze ihrer Mitschüler nicht ertrug ${ }^{(39)}$.

Inwiefern die Weltzeituhr auf dem Berliner Alexanderplatz wirklich ein Treffpunkt der ostdeutschen Jugend war, erfuhren die Lernenden in einem Artikel aus dem westdeutschen Jugendmagazin Scala. Darin berichtete ein junges ostdeutsches Pärchen, dass es sich mit einer 1-Zimmer-Wohnung zufrieden sein musste, weil der Staat größere Wohnungen nur an verheiratete Paare vergab ${ }^{(40)}$. Auch sprach der Artikel den Überwachungsstaat an. Das Lehrbuch Ja, aber... präsentierte somit ein wesentlich realistischeres DDR-Bild als seine Vorgängergenerationen. Die unterschiedlichen Lebenswelten der DDR und der Bundesrepublik waren in den Lehrbüchern angekommen und wurden über literarische und journalistische Texte vermittelt. Über dieses multiperspektivische Vorgehen vermieden die Autoren eine klare Positionierung und boten mehrere Bilder des ostdeutschen sowie des westdeutschen Staates, wobei die völlige Idealisierung der DDR durch eine tendenziell positive Darstellung abgelöst wurde.

Die zwei Gastspiele des Berliner Ensembles in 1950er Jahren wurden im Nachhinein mythologisiert. Colin beschreibt das Phänomen als regelrechte „Brechomanie“. Vgl. Nicole Colın, „Brecht, Bertolt“, in: Dies. u.a. (Hg.), Lexikon der deutsch-französischen Kulturbeziehungen (Anm. 25), S. 141-143 sowie DiEs., „,Das Paradox des politischen Dichters'. Bertolt Brecht im Spannungsfeld der deutsch-deutschfranzösischen Beziehungen“, in: Anne Kwaschiк, Ulrich Pfeil (Hg.), Die DDR in den deutsch-französischen Beziehungen, Brüssel, Peter Lang, 2013, S. 381-397, hier S. 394,

Vgl. J.-C. Duport, Ja, aber... Première Langue 1 (Anm. 32), S. 262.

Vgl. ebd. S. 263. 1949-1990, Köln, Böhlau, 2004, S. 476ff.

Vgl. J.-C. Duport u.a., Ja, aber... Terminales Langue 1 et Langue 2 (Anm. 30), S. 181. 
Dies lässt sich durch die Öffnung der DDR erklären. So bot die kommunistisch orientierte Freundschaftsgesellschaft France-RDA. Association française pour le développement des échanges et de la coopération entre la France et la RDA bereits in den 1970er Jahren auf lokaler Ebene Fahrten in die DDR an. Nach dem Abschluss des Kulturabkommens vom 16. Juni 1980 konnten die zukünftigen Lehrerinnen und Lehrer auch im Rahmen von Universitätspartnerschaften in die DDR reisen ${ }^{(41)}$. Auch wurde das Land von der französischen DDR-Forschung differenzierter dargestellt ${ }^{(42)}$. Nicht zuletzt ist das veränderte Bild eine Folge der westdeutschen Berichterstattung gewesen, die in den 1980er Jahren zu der Überzeugung gekommen war, dass die DDR-Bürger eine eigene nationale Identität gefunden hätten und stolz auf ihr Land seien ${ }^{(43)}$. Die widersprüchlichen Darstellungen in den Lehrbüchern entsprachen schließlich teilweise der Realität des DDR-Alltags, indem sich ein Teil der Bevölkerung eingerichtet hatte: Die Mängel im System waren bekannt und die Unzufriedenheit gärte überall. So lässt sich die Diversifizierung des Bildes durch die veränderten diplomatischen und kulturellen Beziehungen erklären, die sich natürlich mit dem Mauerfall erneut änderten.

\section{Das DDR-Bild in Geschichts- und Deutschlehrwerken zwischen 1990 und 2015}

Die Autorinnen und Autoren französischer Lehrwerke für den Deutschunterricht bemühten sich nach 1990, Nähe zum historischen Subjekt DDR-Bürger herzustellen. Im Gegensatz zu den Geschichtslehrbüchern bieten sie keine zusammenhängende Narration, sondern Anregungen zum Lesen und für Diskussionen sowie Übungen im Sprach- und Landeskundeunterricht. Die Autorinnen und Autoren sind mit ihrer Darstellung nicht auf Kohärenz verpflichtet, so dass das Deutschlandbild weiterhin multiperspektivisch blieb. Anders als in den Geschichtsbüchern steht hier Alltagsgeschichte im Vordergrund, und zwar die Lebensbedingungen „im Schatten der Mauer und des Kalten Krieges [...] in Berlin “(44). Die Kontinuitäten in der DDR-Darstellung zwischen Deutschlehrwerken von vor 1990 und danach sind auffällig. Die Autorengruppen rücken weiterhin Themen wie Musik, Bildung und überhaupt jugendliche Lebenswelten im Sozialismus ins Zentrum. Sie zitieren dieselben Jugendbuchautorinnen und -autoren. Auch die Auszüge aus den Werken nach 1990 schildern die deutschdeutschen Beziehungen als „problematisch“. Lehrbuchverfasserinnen und -verfasser

41 Bei den offiziellen Besuchen und Aufenthalten der Jugendlichen im Rahmen des Programmes „Séjour travail et loisir" wurden die „sozialistischen Errungenschaften“ bei jeder Gelegenheit angepriesen, um die angebliche Überlegenheit der DDR gegenüber der BRD zu demonstrieren. Zugleich hatten die jungen Franzosen dabei aber auch die Gelegenheit, hinter die Kulissen zu schauen. Vgl. F. Flucke, „Land der Dichter und Denker“ (Anm. 1), S.211.

42 Die Unzufriedenheit der Bevölkerung, der repressive Charakter des Regimes und die mangelnde Reisefreiheit erschienen nun auch bei Badia. Vgl. G. EsCHERICH, „Das Bild der DDR in Frankreich“ (Anm. 25), S. 30.

43 Vgl. J. Krauskopf, „Die Darstellung der DDR im französischen Deutschbuch von 1950 bis 1990“, in: Heiner van Bömmel, Herbert Christ, Michael Wendt (Hg.), Lernen und Lehren fremder Sprachen, Tübingen, Gunter Narr, 1992, S. 166-173, hier S. 171.

44 Minna Majlla, Deutschland von außen gesehen. Geschichtliche Inhalte in Deutschlehrbüchern ausgewählter europäischer Länder, Frankfurt am Main, Peter Lang, 2004. 
üben keine explizite Kritik am Gang der Wiedervereinigung, sondern machen diese durch die Textauswahl deutlich ${ }^{(45)}$.

Nach dem Wegfall des Systemkonflikts gab es in Frankreich in den 1990er Jahren im Kontext erinnerungspolitischer Debatten um den Geschichtsunterricht einige Lehrplanänderungen ${ }^{(46)}$. Im Ergebnis wurde politikgeschichtlichen Perspektiven mehr Raum gegeben als sozialgeschichtlichen Themen. Die neuen Lehrpläne gaben zudem vor, dass sich die Schülerinnen und Schüler in der Oberstufe auf das 20. Jahrhundert konzentrieren sollten. Dies war der Rahmen, in dem die Geschichte der DDR vor allem als Ereignisgeschichte neu verortet wurde. Bis zu den ersten überarbeiteten Geschichtslehrplänen von $1995^{(47)}$ galten allerdings weiter sowohl Lehrpläne als auch Schulbücher aus der Zeit des Kalten Krieges, in der die DDR nur sehr marginal und vor allem über den Mauerbau thematisiert wurde. Während der ostdeutsche Staat aus den französischen Geographielehrplänen sofort verschwand, wandelte sich in den Geschichtslehrplänen erst allmählich das Vermittlungsziel. Laut Lehrplan von 1989 beschäftigten sich französische Schülerinnen und Schüler im Geschichtsunterricht der Abiturstufe mit der „sozialistischen Welt“: Politik- und Gesellschaftsgeschichte, aber auch Kultur sollten behandelt werden ${ }^{(48)}$.

Die französischen Geschichtslehrerinnen und -lehrer waren in dieser Zeit gewissermaßen auf sich allein gestellt, was die Modifikation der Inhalte anbelangte. Während der deutsch-französischen Schulbuchtagung in Paris im Dezember 1992 wurde der deutsche Historiker und Schulbuchexperte Rainer Riemenschneider von französischer Seite darum gebeten, „eine kurze Notiz zur Aktualisierung unserer Empfehlungen [des Verbandes an die Lehrerinnen und Lehrer, MMZ] für die Zeit,De 1945 à nos jours" "zu verfassen und an den Geschichts- und Geographielehrerverband APHG (Association des Professeurs d'Histoire et de Géographie) zu schicken. In seiner Antwort warnte er davor, der Darstellung der französischen Medien zu folgen, die vor dem Hintergrund rassistischer Übergriffe wie in Hoyerswerda und Rostock-Lichtenhagen die deutsche Einheit als „deutsche Gefahr“ darstellen würden. Er empfahl schließlich ein Buch ${ }^{(49)}$ zur Lektüre, das die beiden deutschen Staaten mit einer „ostdeutschen Perspektive auf die deutsche Teilung " thematisiert ${ }^{(50)}$.

In den neuen Geschichtslehrplänen von 1995 und 2002 kam DDR-Geschichte nicht explizit vor, lediglich die Situation Berlins als „symbole de la Guerre froide“ wurde auf einer Schulbuchseite zum Mauerbau dargestellt. Die ausgewählten Quellentexte

45 Ebd.

46 Bärbel KunN, „Deutsche Zeitgeschichte in französischen Geschichtsschulbüchern“, in: Susanne PopP und Michael Sauer (Hg.), Zeitgeschichte - Medien - Historische Bildung, Göttingen, Vandenhoeck \& Ruprecht (= Beihefte zur Zeitschrift für Geschichtsdidaktik, Bd. 2), 2010, S. 133-147, hier S. $134 f$.

47 Ministère de l'Éducation nationale, de l'Enseignement supérieur et de la Recherche, Bulletin officiel, $\mathrm{n}^{\circ} 12$ (1995), Histoire et géographie au lycée: programmes.

48 Ebd.

49 Gudula Zückert, Ulrich Zückert, Eine getrennte Geschichte. Die Bundesrepublik Deutschland und die Deutsche Demokratische Republik von 1945/49 bis 1990, Bamberg, Buchner (= Buchners Edition Geschichte, Bd. 2), 1993.

50 „Vision de la division allemande d'un point de vue est-allemand“, NLA - Staatsarchiv Wolfenbüttel, 143 N. Zg. 2009/069, Nr. 174/2. 
zeigten die Sicht der Sowjetunion auf den Mauerbau. DDR-Bürgerinnen und -Bürger als Akteure traten nicht in Erscheinung, die Autorenteams wählten auch keine Dokumente aus Sicht von DDR-Politikern. In Folge des Lehrplans von 2008 behandelten die Schülerinnen und Schüler im Geschichtsunterricht der Troisième den Kalten Krieg im Themenabschnitt „Guerres mondiales et régimes totalitaires“. Darin lautete die Unterrichtseinheit zur DDR wiederum „Berlin au cœur de la Guerre froide“(51). Ein bis zwei Unterrichtsstunden waren dafür vorgesehen. Der Lehrplan beschränkte sich hier auf die Ereignisse Berlin-Blockade, Mauerbau und Mauerfall. Die Schulbücher boten in nahezu identischem Aufbau je zwei bis drei Doppelseiten zum Thema. Berlin geriet als europäische Stadt in den Blick, die im Gegensatz zu Paris im Zentrum der Blockauseinandersetzungen stand. Diese Lehrplanvorgabe lässt sich als Distanzierungsstrategie in Verbindung mit der Aneignung der europäischen Dimension der deutschen Teilungsgeschichte interpretieren. Der Lehrplan für die Première von 2010 sah eine Wiederholung derselben Unterrichtseinheit bei den Klassentypen des allgemeinen Abiturs vor. Die Berliner Mauer blieb im Geschichtsbuch also der Ausgangspunkt der DDRDarstellung. Sowohl für die chronologische Orientierung als auch zur Verdeutlichung des Kalten Krieges sind Mauerbau und -fall wesentliche Ereignisse. In den Geschichtsbüchern wurden zudem viel deutlicher als in den Deutschlehrwerken anklagende Erzählmodi über die DDR dominant und die Mauer diente als solider Zeuge der Interpretation der DDR als „Unrechtsstaat“. Sie erscheint als Symbol der Bipolarität der Welt und als „L'image concrète d'une Europe meurtrie et divisée“(52). In der Erzählung des Triumphs über das Unrecht im Herbst 1989 verkehrt sich der Sinn der Mauer: vom Symbol der Unterdrückung zum Symbol der friedlichen Revolution. Der Mauerfall wird als Etappe dieser Revolution interpretiert. Auf vielen Schulgeschichtsbüchern ist zudem ein Foto des Mauerfalls auf dem Umschlag zu sehen. Die ausgewählten Bilder zeigen die Jubelnden auf der Mauer vor dem Brandenburger Tor und sind ein Code für die deutsche Einheit, aber auch für die Einheit Europas. Sie stellen vermeintlich handelnde Subjekte in den Vordergrund, während die Konfliktlinien unter den Akteuren während der Revolution in der DDR nicht sichtbar sind. Diese Sicht ist vor allem in Westeuropa anschlussfähig. Auf einem Geschichtsbuch von Hatier von 2011 ist die Bildikone der protestierenden Menschen vor der Mauer am Brandenburger Tor in das Plakat „Emprunt de la libération“ integriert ${ }^{(53)}$. Das Plakatmotiv zeigt: Marianne, die französische Nationalfigur, sprengt ihre Ketten. Hier ist die „friedliche Revolution“ eine Aktualisierung der Französischen Revolution, deren Zweihundertjahrfeier bis zum Herbst 1989 kaum zu dem Identität stiftenden Ereignis geworden war, das sich ihre Initiatorinnen und Initiatoren vorgestellt hatten ${ }^{(54)}$. Die Deutschlehrwerke

51 Ministère de l'Éducation nationale, de l'Enseignement supérieur et de la Recherche, Bulletin officiel spécial, nº 6 du 28 août 2008, Programme d'enseignement d'histoire-géographie-éducation civique pour les classes de sixième, de cinquième, de quatrième et de troisième du collège.

52 Pascal ZACHARY u.a., Histoire. Questions pour comprendre le XX $X^{e}$ siècle. $1^{r e} L / E S / S$, Paris, Hachette, 2011.

53 Marianne Boucheret u.a., Histoire $1^{r e}$. Questions pour comprendre le XX $X^{e}$ siècle, Paris, Hatier (= Histoire Lycée, Bd. 1), 2011.

54 Ulrich Pfeil, „Der Bicentenaire, der Fall der Mauer und die Franzosen“, in: Reiner MARCowITz (Hg.), Ein ,neues' Deutschland? Eine deutsch-französische Bilanz 20 Jahre nach der Vereinigung/ 
enthalten ebenfalls teilweise Themenseiten zur Mauer, die technische und historische Daten vermitteln, sowie auf die Propaganda der Mauer als Grenze zum anderen Deutschland eingehen. Erneut finden wir vor allem, wenn auch nicht nur, den Blick von Zeitzeugen sowie Erzählerinnen und Erzählern in literarischen Texten. Durch diese subjektiven Perspektiven rückt sie näher. Sie ist nicht mehr nur ein Bauwerk und Symbol, sondern wird als Grenze im Leben konkreter Personen erfahrbar. Lediglich für die Klassen, die ein Abitur mit literarisch-wirtschaftlichem Schwerpunkt anstreben, gibt es eine zusammenhängende Darstellung der DDR-Geschichte, die in die Tiefe geht. Sie ist eingebettet in eines der vier Jahrgangsthemen der Abiturstufe: „Idéologies et opinions en Europe de la fin du XIX ${ }^{\mathrm{e}}$ siècle à nos jours“. Mit etwa vier bis fünf Unterrichtsstunden findet sich darin der Block „Socialisme, communisme et syn-

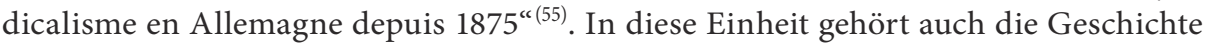
des realexistierenden Sozialismus, parallel zur Geschichte der SPD und der Gewerkschaften in der Bundesrepublik. Dieser Abschnitt ist zwar sehr komplex, kann aber auf dem Vorwissen zum Kalten Krieg aus den jüngeren Jahrgangsstufen aufbauen. Die Schulbücher legen mit ihren Textauszügen zum Leben in der DDR die Interpretation einer "Mitmachdiktatur“ im Sinne Mary Fulbrooks ${ }^{(56)}$ nahe. Im von Vincent Adoumié herausgegebenen Schulgeschichtsbuch von 2012 werden einige Auszüge aus Maxim Leos Familienbiographie Haltet euer Herz bereit zitiert, die zur Identifikation etwa mit der jungen FDJ-Sekretärin Anne anregen, die stolz SED-Mitglied wird ${ }^{(57)}$. In Deutschland wäre eine solche Verortung der DDR-Geschichte zurzeit undenkbar, da die Angst vor einem verharmlosenden Bild von der DDR größer ist.

\section{Deutsch-französische und europäische Perzeptionen im grenzübergreifenden Schulbuch}

Das von Frédéric Delouche herausgegebene Europäische Geschichtsbuch ${ }^{(58)}$, an dem fünfzehn Historikerinnen und Historiker schrieben, unterscheidet sich im Aufbau und in der Erzählperspektive deutlich von französischen und deutschen Geschichtsbüchern. Die Rahmung der Haupterzählung ist die europäische Einigung, identitätsstiftend steht das Buchprojekt unter dem Motto: „Von Europäern für Europäer“.

Erstmalig erschien das Europäische Geschichtsbuch auf Französisch 1992 im Verlag Hachette ${ }^{(59)}$, in einer zweiten überarbeiteten Auflage $1997^{(60)}$. Mehrere europäische Verlage druckten Übersetzungen, die deutschen Ausgaben erschienen 1992 als Schulbuch

Une, nouvelle Allemagne? Un bilan franco-allemand 20 ans après l'unification, München, Oldenbourg, 2010, S. 45-61, hier S. 59f.

55 Ministère de l'Éducation nationale, de l'Enseignement supérieur et de la Recherche, Bulletin officiel, $\mathrm{n}^{\circ} 8$ (2011), Programmes des classes terminales des voies générale et technologique.

56 Vgl. Mary Fulbrook, The People's State. East German Society from Hitler to Honecker, New Haven, Yale University Press, 2008.

57 Vincent Adoumié u.a., Histoire, Paris, Hachette, 2012.

58 Frédéric Delouche u.a., Das europäische Geschichtsbuch. Von den Anfängen bis ins 21. Jahrhundert, Stuttgart, Klett-Cotta, 2012 (3. Aufl.).

59 Frédéric Delouche und Jacques Aldebert, Histoire de l'Europe, Paris, Hachette, 1992.

60 Frédéric Delouche und Jacques Aldebert, Histoire de l'Europe, Paris, Hachette, 1997 (2. Aufl.). 
bei Klett ${ }^{(61)}$ und dann $1998^{(62)}$ und zuletzt $2018^{(63)}$ als Sachbuch bei Klett-Cotta. DDRGeschichte nimmt nur wenig Raum in der Erzählung ein, die auf 460 Seiten die Geschichte Europas „Von den Anfängen bis ins 21. Jahrhundert“ darlegt. In der Ausgabe von 2012 enthalten beide Kapitelauftaktseiten Fotos der Berliner Mauer, die als „das markante Symbol des Kalten Krieges“ noch auf einer weiteren Doppelseite ganzseitig abgedruckt ist. Der nebenstehende Bildtext erläutert das Grenzsystem der DDR. Während das Thema Berliner Mauer also als in die europäische Geschichte integriert dargestellt ist, gibt es keine alltagsgeschichtlichen Einblicke in die Gesellschaft der DDR. Die Autorinnen und Autoren konzentrieren sich auf die Perspektive der sowjetischen Politik in Europa und den gesellschaftlichen Wandel in Westeuropa.

Anders steht es um die Einordnung der DDR in die deutsche und französische Geschichte durch das deutsch-französische Geschichtsbuch Histoire/Geschichte ${ }^{(64)}$, das als erstes binationales Geschichtsbuch für die Oberstufe mit identischer Text-, Bild und Quellenauswahl in den Jahren 2006-2011 erschien. Dem dreibändigen Geschichtsbuch lag ein eigener Lehrplan zu Grunde. Dieser war letztendlich ein Kompromiss aus den gültigen sechzehn deutschen Geschichtslehrplänen und dem französischen Geschichts- und Geographielehrplan. Dadurch, dass ein französischer und ein deutscher Verlag (Nathan und Klett) zusammen arbeiteten, trafen zwei unterschiedliche Funktionsweisen der Schulbuchproduktion und die je national gerahmte Geschichte zweier Gesellschaften aufeinander. Das Geschichtsbuch ist explizit nicht für den bilingualen Geschichtsunterricht konzipiert worden, denn der Aneignung zweier Perspektiven auf ein geschichtliches Thema sollten keine Sprachbarrieren im Weg stehen ${ }^{(65)}$. Im Band Europa und die Welt seit 1945 ist die DDR selbstverständlicher Teil der europäischen Geschichte, nicht aber der Geschichte der deutsch-französischen Beziehungen, die als westdeutsch-französische im Fokus stehen ${ }^{(66)}$. Das Buch ist in fünf Teile gegliedert, zu denen je drei bis vier Kapitel gehören. Elemente einer Geschichte der DDR sind in allen fünf Teilen enthalten. Die Kapitel, in denen die DDR behandelt wird, schrieben zwei westdeutsche Autoren: Ludwig Bernlochner, Autor von Kapiteln zum Kalten Krieg in dem auflagenstarken deutschen Geschichtslehrwerk Geschichte und Geschehen, und Claus Gigl, der ebenfalls schon seit den 1990er Jahren als Schulbuchautor für Klett arbeitete. Unterstützt und beraten wurden sie von einer externen Projektgruppe, einem Comité de pilotage, die aus drei französischen und zwei deutschen

61 Frédéric Delouche, Europäisches Geschichtsbuch, Stuttgart, Klett, 1992.

62 Frédéric Delouche und Jacques Aldebert, Das europäische Geschichtsbuch. Von den Anfängen bis heute, Stuttgart, Klett, 1998 (2. Aufl.).

63 Frédéric Delouche, Das europäische Geschichtsbuch. Von den Anfängen bis ins 21. Jahrhundert, Stuttgart, Klett-Cotta, 2018.

64 Guillaume Le Quintrec und Peter Geiss, Histoire/Geschichte. Europa und die Welt seit 1945. Oberstufe, Paris, Nathan; Stuttgart, Klett, 2006.

65 Rainer Riemenschneider, „Un manuel scolaire peut-il être plurinational? L'exemple du manuel d'histoire franco-allemand“, in: Michèle Verdelhan-Bourgade und Béatrice Bakhouche (Hg.), Les manuels scolaires, miroirs de la nation?, Paris, L'Harmattan, 2007, S. 75-86, hier S. 85.

66 Corine Defrance und Ulrich Pfeil verweisen hier auf die Seite $295 \mathrm{im}$ Schulbuch, Corine DefranCE, Ulrich Pfeil, „Deutsche und Franzosen seit 1945. Vergleichende Herangehensweise als Mehrwert - Zu Teil 5. Dossier Europäische Geschichte à la franco-allemande? Das deutsch-französische Geschichtsbuch in der Analyse“, Dokumente, 2006, S. 82-87, hier S. 82. 
Universitätshistorikern, zahlreichen Ministerialbeamten und - auf der deutschen Seite - einer Lehrerin und einem Schuldirektor bestand. Die DDR-Geschichte ist ausführlicher und anders dargestellt als in französischen Geschichtsbüchern, während sie im Vergleich zu einem deutschen Geschichtsbuch weniger Seiten füllt. Die Rolle Frankreichs im Kalten Krieg beziehungsweise als Besatzer in Deutschland - ein blinder Fleck in den französischen Geschichtsbüchern - wird hier thematisiert. Die verschiedenen Deutungsangebote - die DDR als repressive Diktatur, als „Fürsorgediktatur“(67) und als fortschrittliche Gesellschaft - sind aber trotz weniger Seiten genauso vielseitig wie in einem Geschichtsbuch für die deutsche Oberstufe.

Der französische Verlag Nathan verband das Projekt von Anfang an mit dem Ziel, neue Text- und Bildquellen zu erschließen. Die französische Projektleiterin Delphine Dourlet bewertet das Buch dahingehend als großen, auch nachhaltigen Erfolg. Gerade zum Thema DDR-Geschichte habe man sich auf französischer Seite viel aneignen können ${ }^{(68)}$. Allerdings kommen die drei Bände von Histoire/Geschichte, entgegen der Intention der Herausgeber, fast ausschließlich als Zusatzmaterial und insbesondere in AbiBac-Klassen ${ }^{(69)}$ und im Geschichtsunterricht in der Fremdsprache zum Einsatz ${ }^{(70)}$.

Anhand einer Schulbuchanalyse lassen sich sowohl Eigen- als auch Fremdwahrnehmung der DDR in einer deutschen und französischen Öffentlichkeit herausarbeiten. Zudem zeigt ein Blick auf die Schulbuchmacherinnen und -macher, dass insbesondere zwischen den 1960er Jahren und 1989 ein Austausch zwischen ostdeutschen und französischen sowie westdeutschen und französischen Akteuren stattgefunden hat, der zunächst ein relativ positives DDR-Bild begünstigte. Der Blick auf die DDR als Geschichte hingegen ist viel stärker westeuropäisch geprägt und verweist auf einen breiten deutsch-französischen Konsens bei ihrer Beurteilung. Um dennoch bestehende Differenzen sichtbar zu machen und zu verstehen, sind besonders binationale Projekte wie das deutsch-französische Geschichtsbuch für die Analyse geeignet. Nicht zuletzt bieten vergleichende Schulbuchanalysen das Potential, DDR-Geschichte noch einmal neu zu betrachten und vor allem, sie in größere historische Zusammenhänge einzuordnen.

67 Vgl. Martin SABrow, „Realer Sozialismus als Fürsorgediktatur. Zur begrifflichen Einordnung der DDR“, ApuZ, 20 (1998), S. 34-46.

68 Interview mit Delphine Dourlet, Paris, 2014.

69 Das AbiBac ist ein Verfahren, bei dem das Abitur parallel zum Baccalauréat erworben werden kann.

70 Ilas Körner-Wellershaus, „Mehrsprachigkeit und Schulbuch. Das Deutsch-Französische Geschichtsbuch für die gymnasiale Oberstufe und das Lycée“, in: Annemarie Augschöll BlasBichLER u.a. (Hg.), Mehrsprachigkeit und Schulbuch, Bad Heilbrunn, Klinkhardt, 2013, S. 49-52, hier S. 51; Franziska FluCKe, „Das deutsch-französische Geschichtsbuch. Transnationale Potentiale und nationale Hindernisse in der pädagogischen Praxis“, in: Dorothee Röseberg u. Marie-Therese MÄDER (Hg.), Le Franco-Allemand. Herausforderungen transnationaler Vernetzung/Enjeux des réseaux transnationaux, Berlin, Logos (= Trenn-Striche / Binde-Striche, Bd. 5), 2013, S. 163-178, hier S. $174 \mathrm{f}$. 


\section{Zusammenfassung}

Die Deutsche Demokratische Republik ist Teil der französischen Erinnerung, wie sich anhand einer Lehrbuchanalyse von Geschichts- und Deutschlehrbüchern und ihrer vorhandenen Narrative zeigen lässt. Zunächst reflektierten die Deutschbücher den Wandel der Beziehungen zwischen der DDR und Frankreich und entwarfen insbesondere zwischen den 1960er Jahren und 1989 ein relativ positives DDR-Bild. Nach 1989 wurde die DDR-Geschichte in die Geschichte des Kalten Krieges eingeordnet. Der westeuropäisch geprägte Blick auf die DDR verweist auf einen breiten deutsch-französischen Konsens bei ihrer Beurteilung. Dennoch bestehende Differenzen verdeutlichen binationale Projekte wie das Deutsch-Französische Geschichtsbuch.

\section{Résumé}

Au moyen d'une analyse des manuels scolaires, cet article examine dans quelle mesure la République démocratique allemande fait partie de la mémoire française. Pour cela, nous analyserons les continuités et les ruptures des récits présents dans les manuels d'histoire et d'allemand. Dans un premier temps, les manuels allemands reflètent l'évolution des relations diplomatiques entre la RDA et la France et, surtout entre 1960 et 1989, ils donnent une image relativement positive de la RDA. Puis, après 1989, l'histoire de la RDA fait partie de l'histoire de la Guerre froide. Le regard sur la RDA rejoint celui de l'Europe occidentale et fait apparaître un large consensus franco-allemand. Néanmoins, les différences existantes sont illustrées par des projets binationaux tels que le manuel franco-allemand.

\footnotetext{
Abstract

The GDR is a subject of French textbooks for history and language lessons. Its representation during the existence of the German separation reflects the changing relationship between French intellectuals and politicians and the East-German state. Starting from a negligence of political realities in the GDR, the textbooks vary between the image of a modern social state - influenced by a pro-communist branch of German Studies and a more critical point of view, stressing the lack of basic liberties. After 1990, authors underline the character of the GDR dictatorship in describing the Berlin wall as a symbol of an oppressive regime, which had to be overcome in 1989 by its people. The GDR is taught within the frame of cold war history. The textbook interpretations accord largely with the common Western European memory on the GDR. Remaining differences can be shown in binational textbooks.
} 